\title{
Transformación productiva con equidad: las dimensiones externa y financiera en la propuesta actual de la CEPAL ${ }^{1}$
}

\author{
Ricardo Ffrench-Davis
}

El limitado crecimiento económico de América Latina se ha producido con un aumento de la desigualdad distributiva. Según el autor hay varios espacios de convergencia en el crecimiento con equidad, que al potenciarse contribuyen a lograr esta meta. En este trabajo se describen las propuestas actuales de la CEPAL sustentadas en el enfoque de la transformación productiva con equidad, enfatizando algunos aspectos más relacionados con la dimensión externa. Se describen primeramente algunos valiosos avances que se han llevado a cabo en la región, pero seffalando que aún persisten problemas significativos. En este sentido aborda tres áreas estratégicas: el liderazgo exportador, el sistema financiero interno y las reformas comerciales.

Una pregunta crucial que recorre las discusiones en economía es si es posible crecer con equidad. Yo diría que es difícil crecer sin equidad, aunque también es difícil lograr equidad y crecimiento simultáneamente; sin embargo, hay varios espacios de convergencia entre ambas dimensiones, cuya potenciación facilita la consecución y retroalimentación de ambos objetivos. En el presente artículo expondremos las propuestas actuales de la CEPAL sustentadas en el enfoque de la transformación productiva con equidad, concentrándonos en algunos aspectos más relacionados con la dimensión externa.

La propuesta de la CEPAL busca generar crecimiento económico con equidad. Para ello se requiere "difundir" productividad a través de la sociedad. Cuanto más personas haya que puedan insertarse en la

\footnotetext{
I Texto basado en la Conferencia pronunciada en el Seminario Internacional "Los nuevos desafios en la economla latinoamericana", ILDIS, Quito.
} 
economía con mayor capacidad para producir, podrá haber mayor crecimiento y más equidad. Las personas tienen una dimensión simultánea de productor y consumidor. El apoyo a su carácter de productor deja huellas permanentes: incentivos y oportunidades de educación y capacitación, la adquisición y adaptación de tecnología, el aprendizaje de métodos modernos y funcionales de gestión empresarial, el acceso a nuevos mercados no tradicionales. Todo esto eleva la productividad. Políticas que creen un ambiente propicio para un aumento de la inversión fisica completan un circulo virtuoso, que provee las bases para una transformación productiva con equidad.

\section{Final de la historia ficticia.}

La dinámica de la economía tanto en América Latina como en el resto del mundo tiende a ser actualmente más concentradora de la riqueza que hace 15 ó 20 años. En efecto, si examinamos las situaciones distributivas en EE.UU. o Gran Bretaña y las de distintos países de América Latina veremos que hay un amplio proceso de concentración de la riqueza y de los ingresos. En consecuencia, si el objetivo de la economía es el bienestar de la población -lo cual es una idea medular en esta propuesta-encontramos que esta función no se está cumpliendo de manera satisfactoria, sino que, por el contrario, está aumentando la desigualdad distributiva.

Los elementos determinantes de las tendencias concentradoras que se observan radican en que las políticas económicas se han estado diseñando y ejecutando en base a dos supuestos profundamente erróneos. El primero es que cuando se liberalizan generalizadamente los mercados, de manera lineal y no selectiva, surgen espontáneamente las instituciones que dan oportunidades al alcance de todos. Es decir, se supone que se desarrollan oportunidades y velocidades adecuadas para todos los segmentos de la población: para pobres y ricos, para empresas grandes y pequeñas, para gente de las ciudades y de las áreas rurales, etc.

Sin embargo, la práctica indica que esto no es asi. Persisten muchos mercados incompletos o segmentados. Hay sectores que funcionan muy rảpida y ágilmente, y otros que les toma años ajustarse. Por lo tanto, se crea mucha desigualdad de oportunidades debido a las distintas capacidades de respuesta de los actores sociales y económicos.

El otro supuesto falso es que los mercados que se han liberalizado generalizadamente, generan economías estables y muy dinámicas. Por 
el contrario, to que observamos en los siglos XIX y XX es que la liberalización indiscriminada tiende a generar situaciones cíclicas con grandes altibajos. En los últimos quince años, el mundo tuvo más altibajos que en igual lapso anterior; por ejemplo, en tasas de interés, tipos de cambio y acceso al financiamiento externo. $Y$ el problema es que los ciclos no son neutros para los distintos actores sociales. Los sectores más organizados o modernizados respecto a las operaciones financieras, tienen más capacidad de respuesta. Los sectores más pobres, en cambio, no pueden correr a la misma velocidad que los otros sectores en las fases expansivas, mientras que en los períodos contractivos tampoco pueden "huir" tan rápidamente. Asimismo, los productores de bienes y servicios tienen mayor dificultad para ajustarse que los sectores especulativos. A fin de cuentas, los pobres en comparación con los ricos y los productores versus los especuladores ganan poco con la expansión y pierden mucho en la contracción.

En una serie de publicaciones, la CEPAL ha abordado los temas expuestos. Primero, en 1990, apareció Transformación productiva con equidad, luego, en 1992, Equidad y transformación productiva: un enfoque integrado, enfatizando mucho más la dimensión de equidad, examinando distintos tipos de políticas públicas que tienen un impacto a favor de la equidad y analizando su interrelación con el. desarrollo productivo. Estc enfoque es integrado, en el sentido de que las politicas públicas deben avanzar de manera armónica en ambos planos. En 1995 se publicó Políticas para mejorar la inserción en la economía mundial, siguiendo la linea de Transformación productiva con equidad, pero en esta ocasión enfatizando las relaciones económicas internacionales, donde se discute tanto lo relacionado con los movimientos de capitales como lo referente al intercambio comercial internacional. En esos textos se proponen políticas en el marco de un enfoque integrado, que permitan crecer sostenidamente a través del tiempo, dando más oportunidades a la población para ganarse la vida con mejores ingresos y con más dignidad. $^{2}$

Ultimamente se ha puesto de moda la concepción de que el planeta llegó al "fin de la historia", lo cual supone que habría sólo una manera de hacer las cosas. Esto puede ser cierto en cuanto a que el mercado es una realidad ineludible, pero es muy erróneo en cuanto a que es evidente que no existe una sola forma de construir una economía de mercado, $y$

\footnotetext{
${ }^{2}$ En un anexo, al final de este artículo, se recuentan antecedentes sobre algunas publicacionts seleccionadas de CEPAL más relacionadas con la TPE, y de aportes de sus economistas a la nueva propuesta de CEPAL, incluido su principal inspirador, Femando Fajnzylber.
} 
que ello sea al estilo de lo que se ha llamado "Consenso de Washington". No puede haber un "final de la historia" si el objetivo central de la actividad económica, que es servir a la gente, se está cumpliendo mal. Con retrocesos distributivos y graves problemas de estabilidad y deficiencias en mercados cruciales, estamos al medio de la historia.

La realidad es muy ilustrativa al respecto. En diversos paises hay distintos grados de equidad y de crecimiento, mezclándose un sinnúmero de combinaciones al interior del propio ámbito de la economía de mercado. Esto quiere decir que hay muchos tipos de economía de mercado. A esto también hay que agregar distintos regímenes politicos y diversos grados de democratización.

El modelo de economía de mercado que nos ha llegado "envasado" a América Latina en los últimos años es sólo una de las variantes posibles y que tiene rasgos concentradores por las dos razones principales mencionadas arriba: distribución desigual de oportunidades y propensión a generar economías procíclicas. Eso está reforzado por la constatación de lo ocurrido en los centros de origen de esta variante. En efecto, en las economias estadounidense y británica en los últimos quince años, se ha introducido más elementos de desigualdad que los que tenían antes de los años ochenta. Diversos antecedentes muestran un retroceso distributivo, con un fuerte incremento de la brecha entre ingresos altos y bajos.

En América Latina, por nuestra parte, ha habido un crecimiento limitado (véase el cuadro 1) y la equidad se ha debilitado en estos quince años, lo cual se explica por la naturaleza, secuencia y dosificación de las reformas y las políticas aplicadas. Por ejemplo, los salarios reales se han estancado o deteriorado (véase el cuadro 2), y la brecha entre remuneraciones mayores y menores también se agrandó notoriamente, con un empeoramiento de la distribución del ingreso (véase CEPAL, 1996). Entonces, es necesario examinar lo que deben procurar las políticas públicas, analizando ciertas áreas que nos parecen centrales, principalmente asociadas al sector externo (sesgo resultante de la especialización del autor), en las cuales hay espacio para maniobrar y lograr crecimiento con equidad. 
R. Ffrench-Davis / Transformación productiva con equidad: ...

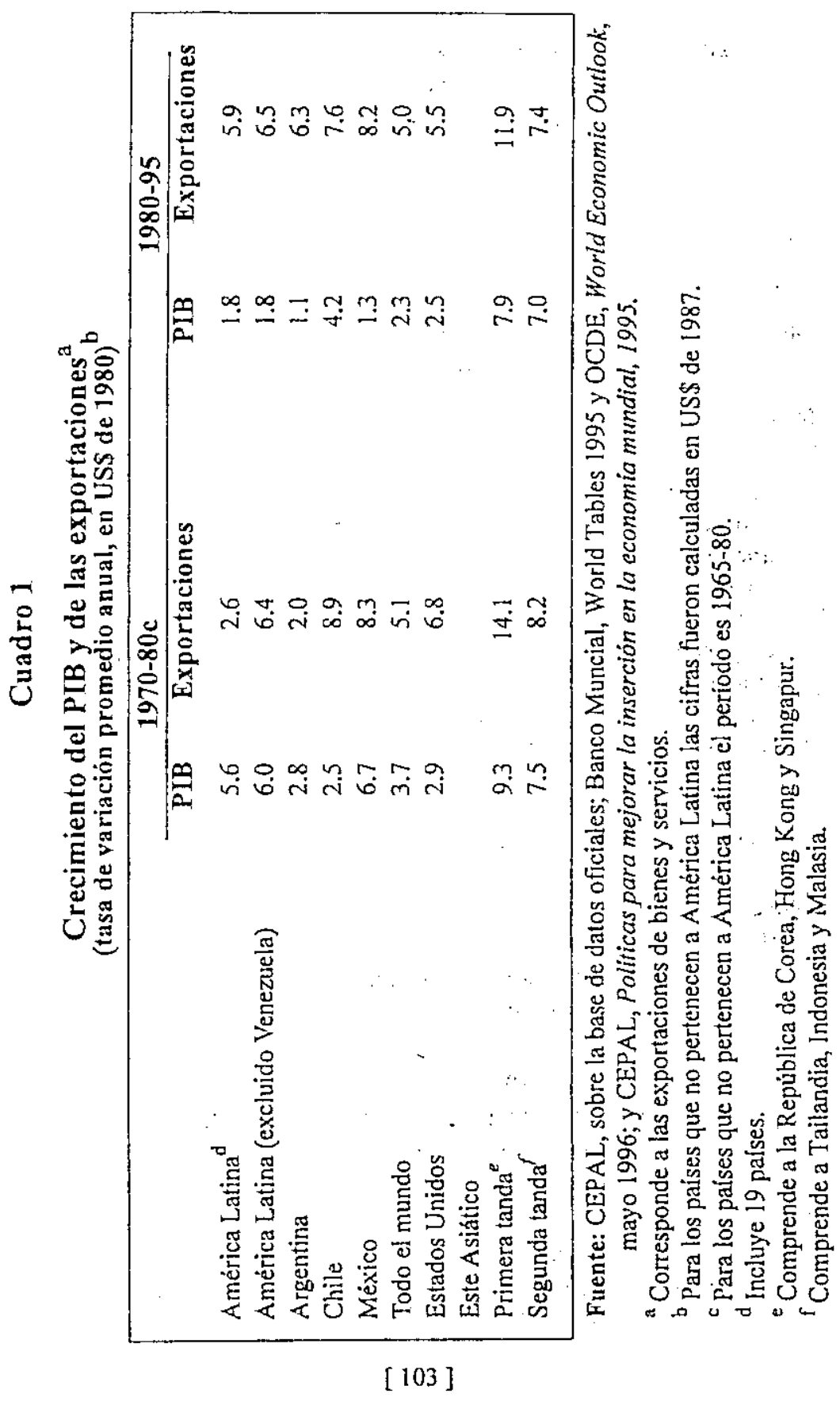


Cuadro 2

\section{América Latina: evolución de las remuneraciones medias reales}

(tasas de variación promedio anual)

\begin{tabular}{lccc}
\hline & $1971-80$ & $1980-90$ & $1990-95$ \\
\hline Argentina $^{\mathrm{a}}$ & -1.2 & -2.6 & 0.2 \\
Brasil $^{\mathrm{b}}$ & 3.9 & 0.6 & -2.5 \\
Chile $^{\mathrm{c}}$ & -3.2 & 0.5 & 4.3 \\
Colombia $^{\mathrm{d}}$ & -0.4 & 1.6 & 0.8 \\
México $^{\mathrm{c}}$ & 1.2 & -2.5 & 2.3 \\
\hline
\end{tabular}

Fuente: CEPAL, sobre la base de datos oficiales.

a Salarios totales medios en la industria manufacturera.

b Salario de trabajadores amparados por la legislación social y laboral, en Río de Janeiro.

${ }^{\varepsilon_{H}}$ Hasta abril de 1993 corresponde a las remuneraciones medias de los asalariados no agricolas. Desde mayo de 1993 corresponde al índice general de remuneraciones por hora.

d Salarios de los obreros en la industria manufacturera.

${ }^{\mathrm{E}}$ Salarios medios en la industria manufacturera.

\section{Avances en América Latina.}

En algunos aspectos, hay avances muy valiosos que deben consolidarse y fortalecerse en América Latina. Por ejemplo, en lo referente a las finanzas públicas. Una familia, un país o una región no pueden gastar más de lo que tienen de manera sistemática, a menos que alguien esté dispuesto a financiarle la diferencia, lo que hoy sólo resulta viable en favor de algunos países muy pobres.

Todos, normalmente, deben encuadrarse dentro de su presupuesto y en eso hay un avance muy sustancial en América Latina. En cuanto a la política fiscal se han logrado avances notables, restableciéndose equilibrios presupuestarios. Las emisiones "inorgánicas" para financiar artificialmente gastos públicos, muchas veces causa de brotes inflacionarios e incluso hiperinflaciones, han perdido vigencia. Ningún pais se beneficia con tasas de inflación de 3 o 4 digitos. Eso resulta muy costoso para 
la gran mayoría de la gente, además de ser un factor enorme de concentración de ingresos por las distintas velocidades a que corren los diferentes sectores sociales, por la capacidad de unos de especular y ganar a costa de otros. Hemos avanzado en la reducción de la inflación, con la mayoría de los países de la región presentando una inflación con tasas de un dígito o del orden de $10 \%$ a $15 \%$ anual.

Otro aspecto positivo es el aumento apreciable del volumen de las exportaciones, lo que es muy positivo porque permite aprovechar lo que sabemos hacer mejor, utilizar las economías de escala, y aumentar la exposicion a mercados más exigentes. Además, en la actualidad estamos exportando una canasta de productos más diversificada; lo que también constituye un elemento positivo, aunque es aún insuficiente. Asimismo, la region ha recuperado el acceso a los mercados de capitales internacionales, especialmente entre 1990 y 1994, en contraste con lo que sucedió en los años ochenta. De otro lado, el producto per cápita, que había caido persistentemente en los años ochenta, se recuperó entre 1991 y 1994.

Todo esto llevó a muchos observadores a ser excesivamente optimistas y creer que no subsisten problemas significativos. Sin embargo, la crisis de México de diciembre de 1994 les hizo poner los pies en la tierra. Los subsecuentes problemas en Argentina, y actualmente en Brasil y' Perú-aunque con distintas escalas e intensidades-reforzaron este "baño de realidad". Esto ha cambiado el ambiente de sobreoptimismo, surgiendo signos de inquietud y diversas interrogantes. En realidad, esto pudo haber surgido antes porque si se analizan los logros y el desarrollo de esas experiencias constataremos que todas ellas tenian ciertas limitaciones o sesgos que conspiraban contra el objetivo de lograr crecimiento con equidad.

Sabemos que en los procesos de ajuste de los años ochenta-los cuales eran necesarios para adecuar el gasto a la capacidad productiva o a la capacidad de financiamiento sostenible-, se cometieron muchos errores. Por ejemplo, los equilibrios fiscales con frecuencia se hicieron a costa de sacrificar la inversión en capital humano o de los salarios de los profesores o funcionarios públicos calificados. También se sacrificó la mantención de la infraestructura vial y portuaria. Todos estos elementos son esenciales para la sustentabilidad de! desarrollo y de los aumentos de productividad los cuales, además, debieran esparcirse en la sociedad con una mejora progresiva de la cantidad $y$, especialmente, de la calidad de la educación. 
En los años noventa, una selectividad errónea ó aún ausencia de ella en lo referente a los movimientos de capitales permitió una excesiva absorción de capitales de corto plazóquełvienenzy se van en enormes cantidades. Esto es muy peligroso porque lós ingresos de capital de corto plazo - asociados a actividades meramente especulativas- son rápidamente reversibles, pudiendo génerar crisis en el sector externo. La experiencia reciente de Argentina y:México ilustra dramáticamente esta situación.

De otro lado, desde inicios de los años ochenta subsiste un bajo nivel general de inversión. Esto es muy perjudicial para el crecimiento y la equidad porque los países no pueden crecer'si invierten poco, necesitando no sólo cantidad sino también calidad en la inversión. Y la calidad va incorporada en los equipos, maquinarias, la educación y capacitación laboral, etc. De esta manera se la incórpora al proceso de producción de bienes y servicios. A su vez, una inversión vigorosa genera crecientes empleos productivos. Por él contrario, la baja tasa de inversión vigente en el mundo, y en particular en América Latina, está determinando que los importantes cambios tecnológicos se traduzcan en el desplazamiento de trabajadores y "desocupación'tecnológica":-

La tasa de inversión, que a médiados de los años setenta era de $24 \%$ a nivel latinoamericano, bajó a $17 \%$ en los ochenta y apenas se recuperó a $19 \%$ en 1994, para bajar el ano siguiente en un punto, debido a la reducción registrada en México y Argentina, principalmente. ${ }^{3}$ De otro lado, el PIB per cápita creció entre 1991 y 1994 en alrededor de $2 \%$, lo cual constituye un progreso apreciable pero claramente insuficiente para reponer el deterioro sufrido durante los años ochenta y muy inferior al patrón exhibido por los países exitosos de Asia. En 1995, la tasa de crecimiento del PIB por habitante fue negativa por las contracciones registradas en México y Argentina; esto refleja las tendencias desestabilizadoras que caracterizan a algunas de las políticas en aplicación.

Con todo, luego de una recuperación en 1996 de $1.7 \%$, el PIB por habitante de América Latina aún era inferior al de 1980.

\section{Tres áreas estratégicas.}

Aquí nos concentraremos en tres áreas estratégicas, que se refieren al liderazgo exportador, al sistema financiero interno, y a las reformas comerciales.

\footnotetext{
${ }^{3}$ Todas las cifras se refieren a la formación bruta de capital fijo, expresadas en dólares de 1980.
} 


\section{a) La estralegia exportadora.}

En lo referente al sector externo y, en particular, a las exportaciones, hay un enfoque que supone simplemente que el crecimiento dinámico de las exportaciones es condición suficiente. Sin embargo, las exportaciones alcanzan sólo al 20\% del PIB mundial (en EE.UU. y en Japón representan sólo el 10\% del PIB); en América Latina alcanzan entre $15 \%$ y $20 \%$ de la producción. Por lo tanto, parece muy limitante suponer que esa fracción genere un crecimiento significativo del total. Otra concepción, más elaborada, plantea que las exportaciones son muy importantes, en cuanto potencialmente involucran un nexo productivo con el exterior, que contribuye a transmitir modernizaciones, innovaciones tecnológicas y métodos de gestión. Se plantea que la tendencia natural es que sea el sector que crezca con mayor rapidez que el resto de la producción. En ese sentido, es un sector líder o de arrastre de la economía.

Al mismo tiempo, este enfoque plantea que, como las exportaciones constituyen sólo una fracción minoritaria del PIB en la gran mayoría de los países, se requiere que esos productos tengan vinculos en el mercadó interno con muchos sectores productores de insumos, servicios, $y$ bienes de capital, lo que define su capacidad de arrastre. Pero, más allá de esos vínculos, las exportaciones por si solas no serán capaces de imprimirle dinamismo al PIB total. Se requerirá también un ambiente propicio para la inversión en productos no exportables.

La insuficiencia de inversión fisica y humana explica que en los años 80 , un vigoroso crecimiento del volumen de exportaciones conviviese con alzas del PIB menores al crecimiento poblacional, y que la mejora en este decenio fuese muy modesta (véase el cuadro 1 y CEPAL, 1995, cap. III).

Lo esencial es que el crecimiento de las exportaciones, efectivamente guíe al $80 \%$ del resto de la producción, pero estimulando la inversión y el empleo en esos sectores. Por muy rápido que sea el crecimiento de las exportaciones, si el resto de la economía crece poco o nada, el bienestar global también aumentará poco. Sin embargo, las exportaciones pueden ayudar mucho a la ecọnomía en su conjunto, dependiendo de su volumen, su estructura, su tasa de crecimiento, sus mercados de destino y la generación de externalidades.

Las exportaciones de América Latina crecieron a una tasa de casi $7 \%$ por año en los últimos años, mientras que el promedio mundial fue de $5 \%$ por año. Pero aqui también es necesario considerar la evolución de los precios de exportación, puesto que América Latina vendió mucho 
más de lo mismo, en un contexto donde la demanda mundial por esos productos crecía poco. Esto es muy importante, ya que los mercados de los productos de exportación evolucionan de manera diferenciada así como las remuneraciones reales de los factores utilizados en su producción. En consecuencia, es una tarea mucho más urgente diversificar las exportaciones, creando nuevas ventajas comparativas para, de este modo, producir bienes y servicios con demandas más dinámicas y poder dar empleo productivo con mejores salarios.

Adicionalmente, si se estimula la difusión de nuevas tecnologías productivas y se impulsa la capacitación laboral, las exportaciones tendrán mayor capacidad de arrastre sobre el resto de la economía.

En cuanto a los mercados de destino de las exportaciones, el comercio intralatinoamericano más que se duplicó en el último quinquenio, estando la mitad de estas exportaciones constituidas por manufacturas y. productos con mayor valor agregado. Esto implica que la canasta exportadora intralatinoamericana es muy distinta a la canasta de exportación hacia el resto del mundo. Gracias a ello, ha tenido un comportamiento de precios más favorable. Entonces, cabe preguntarse por qué se ha mantenido la estructura productiva básica de nuestras economias. Esto se explica porque un quinto del PIB se exporta, y de ello sólo el $22 \%$ se dirige hacia paises de la región, y a su vez, sólo poco más de la mitad implica un mayor valor agregado. Si bien esto constituye un avance, es insuficiente.

En este contexto, los acuerdos del MERCOSUR o del Pacto Andino pueden ayudar en la medida que se cumplan los acuerdos pactados, sin estar permanentemente revisando todo. El desarrollo exige disciplina, seriedad y cumplimiento de la palabra empeñada. Es preferible demorarse más en tomar un acuerdo a condición de que sea más duradero y su evolución futura más predecible. Asi podrá, entonces, estimular más vigorosamente la inversión y los incrementos de productividad.

\section{b) Sistema financiero para el desarrollo.}

De otro lado, el sistema financiero es esencial para estimular el ahorro y canalizarlo hacia el inversionista de largo plazo. También es clave para cerrar los desequilibrios entre ingresos y egresos que cada empresa tiene al no coincidir sus ciclos de producción y venta durante el año. Esto genera periodos de escasez y de abundancia de fondos, que al sistema financiero le corresponde cubrir. 
Sin embargo, nuestros sistemas financieros se han movido principalmente en la dimensión de corto plazo. Como ésta ha sido desproporcionadamente alta, han intermediado el ahorro de algunos hacia el consumo de otros, en lugar de intermediar recursos desde el ahorro hacia la inversión. Esto no ha ayudado a elevar las tasas de ahorro nacional ni los coeficientes de inversión interna que son esenciales para el crecimiento sustentable en el tiempo (Held, 1994).

De hecho a pesar de profundas reformas financieras, la tasa de ahorro de la región ha rondado apenas un $20 \%$ del PIB en comparación con tasas de $30 \%$ o más en el Este asiático. Incluso en los años noventa, la tasa de ahorro bajó significativamente en paises como. Argentina y México (Titelman y Uthoff, 1997).

El deficiente desempeño del ahorro $y$ la inversión es un rasgo distintivo en América Latina que, junto con las tasas de interés reales tan inestables y generalizadamente altas, constituyen serias distorsiones que se deben corregir. En la región hubo tasas reales de interés bajas o negativas hasta los años setenta. Luego aumentaron en exceso, al pasarse de una política de intervención extrema a una excesivamente liberalizante.

La experiencia de Asia oriental, en cambio, no tiene esos rasgos. Las reformas financieras en varios paises europeos tampoco tuvieron esas características. En América Latina el sistema se liberalizó de manera indiscriminada y abrupta, en un contexto de superintendencias y esquemas regulatorios muy débiles. De este modo, muchos bancos son captados por ciertos grupos, los cuales se prestan dinero a sí mismos, dejando pocos recursos disponibles para el resto de agentes.

Esos grupos tienen que competir por préstamos a tasas de interés măs altas. $\mathrm{Y}$ cuando los créditos son de muy corto plazo, puede entenderse que los deudores puedan comprometerse a pagar tasas de interés tan elevadas como las que han regido en años recientes en muchos países. En efecto, $3 \%$ de interés a 30 dias parece más fácil de pagar que $40 \%$ anual; pero la realidad al final es lo último. Entonces, los problemas de la mala calidad de las carteras, el excesivo autopréstamo y los mercados de tan corto plazo - a causa de la ausencia de regulación y la debilidad de las superintendencias-son algunos de los causantes de los problemas en el ámbito financiero.

Las instituciones financieras de las economías desarrolladas y varios países de Asia Oriental han creado segmentos dé créditos de largo plazo. Sin embargo, éstos no surgieron espontáneamente, sino que fueron estimulados por los bancos centrales o ministerios de hacienda. En paises 
como Japón, por ejemplo, el mercado de capitales fue fundamentalmente de largo plazo y muy pocode corto plazo hasta mediados de los ochenta: Esto plantea una tarea imprescindible: operar una reforma al interior del propio proceso de reforma, es decir, reformar las reformas, para desarollar los segmentos, ausentes o incompletos.

Se suscribió en demasia el camino del Cono Sur de los años setenta. No obstante, los tres países de esa' zona que aplicaron reformas neoliberales radicales, sufrieron en los años setenta u ochenta crisis bancarias generadas por malas carteras, con tasas de interés tremendamente altas y.que segulán abultándose. Naturalmente, cuando llegó el momento de cobrar, pocos podian pagar. $Y$ es que en general la empresa, por eficiente que sea, no está en condiciones de pagar tasas de interés real de $20 \%$ o $40 \%$. Entonces, los bancos tenían carteras ficticias, en un contexto de superintendencias muy débiles ' $y^{\prime} \cdot \sin$ normativas adecuadas para la evaluación de activos y pasivos. Esto plantea una tarea central, consistente en "completar el mercado". Es decir, que haya segmentos que den créditos de largo plazo bien evaluados, que mejore el acceso de la PYME, y.que el manejo de las tasas de interés sea más consistente con la productividad efectiva de las empresas.

\section{c) Reformas comerciales y desarrollo productivo.}

Las reformas comerciales en América Latina en los años noventa se implementaron con rebajas arancelarias sustanciales, a menudo unilaterales, que se hicieron en un contexto donde varios países de la región se encontrában en situaciones relativamente recesivas y con tasas de interés muy altas. Paradójicamente, en lugar de registrarse devaluaciones cambiarias (encarecimiento del dólar con relación a las monedas nacionales), como teóricamente sucedería cuando se liberalizan las importaciones, aconteció lo contrario. Estas apreciaciones cambiarias estuvieron asociadas a los excesivos ingresos de capital, cuando la règión pasó de la gran escasez de fondos externos de los ochenta, a la abundancia de los inicios de los noventa. Asi, de los 18 principalés paises de América Latina, 13 experimentaron apreciaciones cambiarias muy significativas entre 1990 y 1994.

Todo esto generó un ambiente que dificultó tremendamente el proceso de ajuste de las empresas sustituidoras de importaciones, las que súbitamente sévieron enfrentadas a un triple shock adverso, asociado a la significativa reducción de la protección con lo cual se abaratan los productos importados, a la apreciación cambiaria que abarata aún más a 
estos productos, y a las elevadas tasas de interés que les encarecía el crédito necesario para financiar su reconversión productiva. Observando todos los sectores, el resultado fue que, si bien se registró un incremento persistente de las exportaciones, paralelamente se operó un proceso mucho más intenso de desustitución de importaciones, donde las empresas que no pudieron ajustarse a las nuevas condiciones simplemente quebraron. Algunos no justificaban su existencia, pero otras quebraron por un mal manejo macroeconómico.

La teoría simple supone que todos los recursos liberados por las empresas que quiebran (trabajo, capital, conocimiento) serán absorbidos por los sectores exportadores. Sin embargo, si las exportaciones crecen más lentamente que la desustitución de importaciones, el desempleo termina aumentando. Esto significa, como lo muestra la evidencia, que durante varios años hay más inversionistas castigados que premiados. Este es el costo de la manera cómo se implementó la reforma comercial en la región, y es uno de los factores que explica la baja tasa de inversión y la escasa creación de empleos productivos en los años recientes.

\section{Una síntesis.}

Es entonces fundamental y urgente facilitar el proceso de reconversión productiva, creando un ambiente macroeconómico adecuado. En tal sentido, algunos elementos centrales son la reforma financiera, que facilite la canalización de recursos a tasas de interés razonables, a plazos más largos y con mayor equidad en las oportunidades de acceso al financiamiento; la regulación del movimiento de capitales, para moderar el exceso de capitales de corto plazo y especulativos, de modo de evitar la inestabilidad y la vulnerabilidad de las economias nacionales; y la política cambiaria que permita mantener un tipo de cambio real sustentable que estimule las exportaciones a través del tiempo. Todos estos son aspectos que ayudan mucho a la reconversión, mejorando la distribución de oportunidades, orientando a los empresarios respecto de las ramas productivas donde pueden invertir y permitiendo que los trabajadores que fueron expulsados de un sector puedan ser absorbidos por otro sector. Evidentemente, lo ideal es que primero ocurra esta absorción y no al revés, porque eso tiene mucho impacto sobre los niveles salariales y sobre la productividad global. Cuando esta absorción es más rápida, también habría menos desempleados, menos subutilización de la capacidad productiva y menos fábricas cerradas. 
Hay otras medidas más específicas, como las políticas de conversión : sectorial en el ámbito agrícola o manufacturero. Sin embargo; es esencial tener presente que es muy fácil malgastar recursos (tari "escasos en nuestros países) tratando de mantener a la gente en empresas y sectores moribundos. Más bieñ lo que debemos hacér!es crear nuevas oportunidadès de empleo," lo cual requiere reconversióni de empresärios, trabajadores y equipos; capacitación laboral; fuentes de finâñáiamiento; $\mathrm{y}$ un ambiente macroeconómico estable, que estimule la inversióń productiva. Esto exige distinguir entre las empresas moribundas y las que pueden efectivamente reconvertirse, ya sea en el mismo sector o en actividades alternativas.

Tener crecimiento económico no es fäcil; tampoco conseguir equidad. En el 'mundo son excepcionales las experiencias que presentan crecimiento sostenido y equidad. Lo más común es que ambós brillen por su ausencia. Es lo que Fernando Fajnzylber llamó:"él ccasillero vacío". Sin embargo, es posible progresar en elllenado de ese casillero, a la luz de lo avanzado en la región en los 40 años anteriorès coñ la sustitución de importaciones, a pesar de los errores voluntaristas; por lo que avanzamos en la educación, por lo que hemos avanzado -aunque con altibajos- en el proceso de democratización y participación; por lo que se ha aprendido en los años recientes en dar mayor espacio al mercado, en especial en el ámbito microeconómico; por el aprendizaje en el manejo macroeconómico por el número creciente de empresas que han aprendido a incursionar en los mercados externos y a exportar; por los recursos naturales que son utilizables y reconvertibles en productos con mayor valor agregado, de modo de poder colocarlos en mercados más favorables.

Entonces, tenemos un espacio promisorio que requiere disciplina, organización y mucho pragmatismo. Esto involucra a todas las reformas. Algunos piensan que la liberalización comercial radical y generalizada era el camino correcto. En realidad, hay que senalar que muchas de las restricciones al comercio eran exageradas y absurdas. Sin embargo, en muchos casos se requería avanzar gradualmente y de manera armónica e integral, cautelando que las velocidades con que ciertos sectores se contraían y otros se expandian, fueran consistentes entre sí. Esto era indispensable para facilitar el traslado de recursos de unos sectores a otros, y evitar así situaciones recesivas, con su secuela de desempleo, desperdicio de recursos y empeoramiento de la equidad.

En tal sentido, hay que destacar que hay tareas pendientes que debemos realizar los paises latinoamericanos respecto de completar 
mercados y crear o fortalecer instituciones. Al respecto, mucho tenemos que aprender del pragmatismo del Este asiático. En relación a los propios países de la región, en particular cabe intensificar el intercambio comercial entre nuestros países, dado que la canasta exportadora intrarregional tiene la virtud de abarcar productos más elaborados, con un mayor valor agregado y mayores externalidades. Los progresos en MERCOSUR son muy esperanzadores.

Por último, en cuanto al rol de los consensos sociales, hay muchos espacios de convergencia entre sectores políticos y sociales distintos. Hay mucho camino por recorrer, pero si pretendemos imponer el cien por ciento de nuestros propios postulados, lo más probable es que no logremos avanzar nada $o$ aún retrocedamos. Entonces, es necesario buscar espacios de encuentro en los cuales el conjunto de sectores estemos dispuestos a avanzar en la construcción de consensos sociales. Esto les va señalar a los distintos actores económicos participantes (gobiernos, empresarios y trabajadores), los senderos probables en los cuales nos vamos a dirigir durante los próximos años. En tal sentido, el desarrollo con equidad requiere mucha labor técnica para, a partir de allí, se promuevan las propuestas en el mundo de las decisiones politicas.

\section{ANEXO}

El artículo pionero, que abre la linea de reflexión intelectual sobre transformación productiva con equidad, es el informe del mismo nombre, coordinado por Fernando Fajnzylber, publicado en 1990. Luego se avanzó en el examen de políticas económicas alternativas en El enfoque integrado, informe coordinado por Joseph Ramos. Diversos tópicos más específicos se har ábordado en sucesivos documentos de la CEPAL. Entre ellos, cabe destacar el informe respecto del rol, diagnóstico y propuestas, sobre educación y conocimiento, con la participación activa de Fernando Fajnzylber, Martine Guerguil y Ernesto Ottone, publicado en 1992, seguido por la reflexión enfocada en la integración, presentando la propuesta de Regionalismo abierto, dirigida por Gert Rosenthal, publicado en 1994, y el informe integrado dirigido a examinar la inserción comercial y financiera externa, Políticas para mejorar la inserción en la economía mundial, con propuestas alternativas de políticas 
económicas, publicado en 1995 (ahora en reedición actualizada para el Fondo de Cultura Económica en 1997). En este informe (así como en los anteriores) se contó con la activa participación de un amplio grupo de especialistas de la CEPAL y de colaboradores externos. Profundizaciones de las propuestas generales se encuentran, por ejemplo, en varios trabajos pioneros de Fernando Fajnzylber, original impulsor de los planteamientos de CEPAL (en especial véase el artículo sobre "El casillero vacío"); y en publicaciones sobre desarrollo tecnológico, dirigidas por Jorge Katz; y en estudios sobre la dimensión financiera con especialistas como Carlos Massad, Robert Devlin, Gunther Held y Roberto Zahler. Como siempre sucede en instituciones intensas en creaciones colectivas, habremos cometido muchas injustas omisiones. Síntesis de las propuestas aparecen en diversos textos de Gert Rosenthal, por ejemplo en Rosenthal (1994), en Ramos (1994 y 1995), Lahera, Ottone y Rosales (1995), y Ottone (1996).

\section{Referencias Bibliográficas}

CEPAL, Transformación productiva con equidad. La tarea prioritaria del desarrollo de América Latina y el Caribe en los años noventa, Publicación de las Naciones Unidas. $N^{\circ}$ de venta: S.90.II.G.6., Santiago, marzo, 1990.

-.. - Equidad y transformación productiva: un enfoque integrado, Publicación de las Naciones Unidas. $N^{\circ}$ de venta: S.92.11.G.5., Santiago, abril, 1992.

- - "El regionalismo abierto en América Latina y el Caribe: la integración económica al servicio de la transformación productiva con equidad", Serie de libros de la CEPAL 39, Santiago, 1994.

- - América Latina y el Caribe: politicas para mejorar la inserción en la economia mundial, Publicación de las Naciones Unidas, $\mathrm{N}^{\circ}$ de venta: S.95.II.G.6., Santiago, abril, 1995.

-- Panorama social de América Latina y el Caribe, 1996, Santiago, 1996.

Fajnzylber, F., 'Industrialización en América Latina: de la 'caja negra' a! "casillero vacio'", Cuaderno de la CEPAL, $\mathrm{N}^{\circ} 60$, Publicación de las Naciones Unidas, $N^{\circ}$ de venta: S.89,II.G.5., Santiago, 1990. 
Ffrench-Davis, R. y S. Griffith-Jones (comps.), Las nuevas corrientes financieras hacia la América Latina: fuentes, efectos y politicas. México, D.F. y Santiago: CEPAL/Fondo de Cultura Económica, 1995.

Held, G., "Liberalización o desarrollo financiero?", Revista de la CEPAL, N54, Santiago, diciembre, 1994.

Katz, J. (ed.), Estabilización macroeconómica, reforma estructural y comportamiento industrial. Buenos Aires: CEPAL/Alianza Editorial, 1996.

Lahera, E., E. Ottone y O. Rosales, "Una sintesis de la propuesta de la CEPAL", Revista de la CEPAL, N55, Santiago, abril, 1995.

Ottone, E., "Transformación productiva con equidad: surgimiento, significado y desarrollo de una propuesta", mimeo, 1996.

Ramos, J., "Sintesis del planteamiento de la CEPAL sobre la equidad y transformación productiva", Desarrollo Productivo, $N^{\circ} 17$, CEPAL, Santiago, 1994.

...- "Es posible crecer con equidad?", Revista de la CEPAL, N56, Santiago, agosto, 1995.

Rosenthal, G., "Reflexiones sobre el pensamiento económico de la CEPAL", Revista Pensamiento Iberoamericano, N²4-25, Madrid, 1994.

Sunkel, 0 . (ed.) El desarrollo desde dentro: un enfoque neoestructuralista para la América Latina. México, D.F.: Fondo de Cultura Económica, 1991.

Uthoff, A. y D. Titelman, "The relationship between foreign and national savings under financial liberalization", mayo, 1997. 